\title{
Rejection thresholds (RjT) of sweet likers and dislikers
}

\section{Article}

\section{Accepted Version}

Creative Commons: Attribution-Noncommercial-No Derivative Works 4.0

Methven, L., Xiao, C., Cai, M. and Prescott, J. (2016) Rejection thresholds (RjT) of sweet likers and dislikers. Food Quality and Preference, 52. pp. 74-80. ISSN 0950-3293 doi: https://doi.org/10.1016/j.foodqual.2016.03.012 Available at https://centaur.reading.ac.uk/61851/

It is advisable to refer to the publisher's version if you intend to cite from the work. See Guidance on citing.

To link to this article DOI: http://dx.doi.org/10.1016/j.foodqual.2016.03.012 Publisher: Elsevier

All outputs in CentAUR are protected by Intellectual Property Rights law, including copyright law. Copyright and IPR is retained by the creators or other copyright holders. Terms and conditions for use of this material are defined in the End User Agreement.

\section{www.reading.ac.uk/centaur}

\section{CentAUR}

Central Archive at the University of Reading

Reading's research outputs online 
1 Rejection Thresholds (RjT) of Sweet Likers and Dislikers

2

L. Methven ${ }^{a^{*}}$, C. Xiao ${ }^{a}$, M. Cai ${ }^{a}$, J. Prescott ${ }^{b}$

4

5 a Department of Food and Nutritional Sciences, University of Reading, Whiteknights,

$6 \quad$ Reading RG6 6AP, UK

$7 \quad{ }^{b}$ TasteMatters Research \& Consulting, Sydney, Australia

$8 \quad{ }^{*}$ Corresponding author. E-mail address: I.methven@reading.ac.uk 


\section{Abstract}

Sweetness is generally a desirable taste, however consumers can be grouped into sweet likers and dislikers according to optimally preferred sucrose concentrations. Understanding the levels of sweetness in products that are acceptable and unacceptable to both consumer groups is important to product development and for influencing dietary habits. The concentrations at which sucrose decreases liking (the rejection threshold; $\mathrm{RjT}$ ) in liquid and semi-solid matrices were investigated in this study. Thirty six consumers rated their liking of 5 sucrose aqueous solutions; this identified 36\% sweet likers (SL) whose liking ratings increased with increasing sucrose and $64 \%$ sweet dislikers (SD) whose liking ratings decreased above $6 \%$ $(w / v)$ sucrose. We hypothesized that SL and SD would have different RjT for sucrose in products. This was tested by preparing 8 levels of sucrose in orange juice and orange jelly and presenting each against the lowest level in forced choice preference tests. In orange juice, as sucrose increased from $33 \mathrm{~g} / \mathrm{L}$ to $75 \mathrm{~g} / \mathrm{L}$ the proportion of people preferring the sweeter sample increased in both groups. However, at higher sucrose levels, the proportion of consumers preferring the sweet sample decreased. For SD, a RjT was reached at $380 \mathrm{~g} / \mathrm{L}$, whereas a significant $\mathrm{RjT}$ for $\mathrm{SL}$ was not reached. RjT in jelly were not reached as the sweetness in orange jelly was significantly lower than for orange juice $(p<0.001)$. Despite statistically significant differences in rated sweetness between $S L$ and $S D(p=0.019)$, the extent of difference between the two groups was minor. The results implied that sweet liker status was not substantially related to differences in sweetness perception. Selfreported dietary intake of carbohydrate, sugars and sucrose were not significantly affected by sweet liker status. However the failure to find an effect may be due to the small sample size and future studies within a larger, more representative population sample are justifiable from the results of this study.

\section{Highlights}

- In orange juice preference increased as sucrose was increased from $33 \mathrm{~g} / \mathrm{L}$ to $75 \mathrm{~g} / \mathrm{L}$

- In orange juice the sucrose rejection threshold for sweet dislikers was $380 \mathrm{~g} / \mathrm{L}$

- Rejection threshold for sweet likers were higher than for sweet dislikers

- Sweetness intensity was significantly lower in orange jelly than juice 
- Sweet liker status was not substantially related to sweetness perception

\section{Keywords}

Sweet liking, rejection thresholds, perceived intensity

\section{Introduction}

Humans have an innate liking for sweetness (Drewnowski, Mennella, Johnson, \& Bellisle, 2012) as reflected in positive facial expressions even in newborn infants (Berridge, 2003). However, variation in individual liking of sweet taste has been reported since the 1970s (Lundgren et al., 1978; Pangborn, 1970) and recent studies have classified people to be either sweet likers (SL) or sweet dislikers (SD) based on their hedonic responses to sucrose solutions (Holt, Cobiac, Beaumont-Smith, Easton, \& Best, 2000; Ji-Yoon, Prescott, \& Kwang-Ok, 2014; Kim, Prescott, \& Kim, 2014; Mennella, Lukasewycz, Griffith, \& Beauchamp, 2011; Yeomans, Prescott, \& Gould, 2009). The practical implications of this distinction have yet to be explored in detail. It might be expected that sweet liking would be associated with increased preference for, or consumption of, sweet foods. However, definitive evidence for this is lacking. Mennella et al. (2011) reported an association between preferred concentrations of sugar solutions and the sugar content of preferred breakfast cereals. Another recent study found that SL gave significantly higher liking scores to listed sweet foods than did SD; and in tasting milk and dark chocolate the SL had a significantly greater preference for the sweeter milk chocolate (Kim, et al., 2014).

As humans have an innate liking for sweetness, the term "sweet dislikers" is somewhat inaccurate. SD are unlikely to dislike sweetness in totality, but merely prefer moderate sweetness levels to high sweetness levels. Therefore, another possibility is that SL and SD may differ in their tolerance for variations in the sweetness of foods. Thus, we might expect that optimal liking for the sweetness of foods or beverages would be reached, and exceeded, at lower sweetener concentrations for sweet dislikers than for sweet likers. One approach to evaluating such differences is to measure the point at which a food or beverage is rejected when a quality (in this case, sweetness) is increased. Such rejection thresholds ( $R \mathrm{KT}$; also known as consumer RjT) have previously been determined for tastes and flavours that might be expected to adversely affect acceptability. These have 
included 2,4,6-trichloroanisole in wine (TCA, cork taint) (Prescott, Norris, Kunst, \& Kim, 2005), 1,8-cineole (eucalyptol) in wine (Saliba, Bullock, \& Hardie, 2009), bitterness and astringency in wines spiked with catechin-rich extracts (Yoo, Saliba, Prenzler, \& Ryan, 2012), added bitterness in chocolate (Harwood, Ziegler, \& Hayes, 2012b) and polyphenols in chocolate (Harwood, Ziegler, \& Hayes, 2013). In each case, these studies determined the lowest concentration of the compound that became objectionable in a specific food/beverage matrix. The methods to detect RjT were simple and similar in each study. In the first paper concentrations of TCA were added to eight wine samples (Prescott et al., 2005) and every TCA-spiked wine was compared to a sample of control wine in forced-choice preference tests. In three chocolate studies (Harwood, Loquasto, Roberts, Ziegler, \& Hayes, 2013; Harwood, Ziegler, \& Hayes, 2012a; Harwood et al., 2012b), participants were grouped as selfreported milk or dark chocolate likers, which is perhaps analogous to bitter dislikers/ likers. The papers reported different RjT for bitterness in chocolate milk, chocolate ice cream and in a solid chocolate coating between these two groups.

Here we investigate whether RjT can be determined for sweetness in both liquid and semi-solid food formats (orange juice and orange jelly) and consider whether sweet RjT may vary as a function of SL/SD status, a classification based on responses to sucrose solutions. One limitation of this approach might be that liking of sweetness in aqueous sugar solutions does not predict liking nor rejection of sweetness in a more complex food matrix, where food format and presence of other tastants can suppress sweetness. However previous studies have shown a relationship between liking for sweetness in solution and liking of sweetness in foods (Mennella et al.,2011; Kim, et al., 2014), hence justifying investigation of sucrose RjT by SL/SD in the present study.

Differences in taste sensitivity have been associated with differences in tastant liking, where higher sensitivity tend to lead to reduced liking at high tastant levels (Hayes \& Duffy, 2008). However, early studies on sweet perception and liking do not find such a relationship. A paper in 1978 concluded that whereas children and adults perceived sweetness in a similar manner their hedonic responses were substantially different (Moskowitz, 1978). In the same year a study of sweetness in coffee found that sensitivity to sweetness in coffee was not related to differences in liking for sucrose 
level (Lundgren et al., 1978). Any link between liking of sweet taste, sweet taste sensitivity, intake of sweet foods and body mass index (BMI) remains controversial. Bartoshuk, Duffy, Hayes, Moskowitz, and Snyder (2006) criticised many studies for using scales with intensity labels that assume the same absolute intensity is perceived by all; they proposed that the intensities denoted by labels vary depending on the participants experience of the tastant. To address this, they used the general labelled magnitude scale (gLMS) to measure perception of, and liking for, both of sweet and fat, in 3740 US subjects with a BMI range of $<18.5$ to 50 . They found that obese subjects experienced reduced sweetness and liked both sweet and fat more than non-obese subjects. In contrast, however, a recent study also using gLMS scales found no relationship between sweetness ratings and either dietary intake of sugars or BMI, although the study sample was much smaller and narrower in BMI range (Cicerale, Riddell, \& Keast, 2012). Therefore, to further increase data in this area we collected sweetness perception data to determine whether there was a relationship between sweet liking and sweetness perception. As a secondary output measure, dietary intake was also measured in order to investigate any relationship between self-reported sugar intake and either sweet liker status or sweet perception.

The hypotheses of this study are: (1) The RjT method developed for objectionable flavours is able to define RjT of added tastants that vary in desirability; (2) SL have a higher RjT for sweetness than SD; (3) Low RjT for sweet taste are associated with greater sweetness; (4) sweetness and RjT for sweetness will differ in absolute sucrose concentration within liquid and semi-solid food matrices, and (4) Dietary intake of sugars will be higher for SL. In order to investigate these hypotheses, the study objectives were to first classify SL and SD based on liking ratings for sucrose solutions, analyse RjT of sweetness in orange juice and orange jelly using forced choice preference tests, determine sweetness intensities in orange juice and jelly, and investigate any relationship between sweet liker status and dietary intake of sugars.

\section{Method}

\subsection{Subjects}

Thirty-six non-smokers, age 18 to 50 years, with no relevant food allergies, recruited from the University of Reading (Table 1), provided informed written consent. The 
study was approved by University of Reading Ethics Committee (study number 03_13). The subjects had a median age of 26 and there were a higher proportion of females $(66 \%)$ than males (34\%). The median BMI was within the normal weight range at $22 \mathrm{~kg} / \mathrm{m}^{2}$.

\subsection{Sensory stimuli}

The subjects were required to complete three tests: (1) Liking of sucrose solutions using visual analogue scales (VAS) to establish SL and SD classifications; (2) Rejection Thresholds (RjT) of sucrose in orange juice and orange jelly using paired preference tests and (3) Sweetness intensity measurements in the juice and jelly samples using labelled magnitude scales (LMS).

The taste stimuli used in the SL/SD determination were five aqueous solutions of sucrose (Tate \& Lyle, UK) (3\% w/v, 6\% w/v, 12\% w/v, 24\% w/v and $36 \% \mathrm{w} / \mathrm{v})$. The sucrose was dissolved in mineral water (Harrogate Spa, UK). Orange juices and jellies with sucrose additions in an increasing geometric progression of ratio 1.5 were prepared for RjT and LMS tests. The eight levels of sugar in orange juice were L1 (33.3g/L), L2 (50.0g/L), L3 (75.0 g/L), L4 (112 g/L), L5 (169 g/L), L6 (253 g/L), L7 $(380 \mathrm{~g} / \mathrm{L})$ and L8 (569 g/L). A mixture of $100 \mathrm{ml}$ orange juice (Tropicana Smooth, PepsiCo, UK) containing 100 g/L sugar and $200 \mathrm{ml}$ mineral water (Harrogate Spa, Harrogate, UK) was used to achieve the L1 juice sample. L2 to L8 juice sample were achieved by adding the required additional amount of sucrose to L1 juice sample and heating to $40( \pm 4)^{\circ} \mathrm{C}$ to ensure the sugar was fully dissolved. The L1 sample was also heated to $40( \pm 4)^{\circ} \mathrm{C}$ for consistency.

As it was expected that the sweetness of the jelly samples would be lower than in the juice, the eight sucrose additions to the orange jellies started higher at L2 $(50.0 \mathrm{~g} / \mathrm{L})$, levels 3 to 8 were the same as in juice and one higher addition level was prepared, L9 (854 g/L). To produce jelly samples, vegetarian gelling powder (50 g/L) (Asda, UK) was added to juice samples with the designated sucrose additions. The ingredients of vegetarian gelling powder were the gelling agent, agar, and maltodextrin. Samples were heated to boiling for 1 minute in order to dissolve the agar, then cooled to room temperature and held refrigerated $\left(4^{\circ} \mathrm{C}\right)$ overnight. All samples were labeled with random three digit codes. 


\subsection{Procedure}

Participants completed the sweet liker status test and RjT tests in their first visit. In their second visit, at least 1 week later, sweet intensities were measured. Of the 36 participants in visit 1, two withdrew from the study at visit 2; however their data from visit 1 were retained. The VAS scale used for the sweet liker test $(15 \mathrm{~cm}$, scaled 0 to 100) was marked with a neutral point at half scale length and had end-anchors from "Extremely unpleasant" to "Extremely pleasant". This VAS scale has previously been used to classify SL and SD (Holt et al., 2000). The five sucrose samples were served to each participant monadically, in a balanced presentation order.

The RjT test was a forced choice test based on the method by (Prescott et al., 2005).

In the first visit, weights and heights of participants were measured and used to calculate BMI. Additionally, participants were asked to fill in a Food Frequency Questionnaire (FFQ) as used by the European Prospective Investigation into Cancer and Nutrition (EPIC) group (EPIC, 2015).

To measure sweetness intensity of the jelly and juice samples in visit 2, an LMS scale was used with six verbal descriptors ranging from "barely detectable" to "strongest imaginable" positioned in a logarithmic manner on a vertical line. The eight sucrose levels in jelly and juice samples were presented monadically in a 
balanced order.

All sensory tests were carried in individual booths with red lights and at a fixed room and product temperature $\left(23 \pm 2^{\circ} \mathrm{C}\right)$. Between samples, participants had 1 minute to cleanse their palate with filtered water and crackers (Carrs Water Biscuits, United Biscuits, UK). Compusense five software (version 5.2.19, Ontario, Canada) was used for data collection.

\subsection{Data analysis}

Significant differences in VAS scale liking ratings of the five aqueous sucrose samples were analyzed by ANOVA. SL and SD were determined by two methods. The first used agglomerative hierarchical clustering (AHC) using Ward's method, dissimilarity and truncated at 2 classes. In order to compare results with previous literature, this was compared to an earlier method where individuals average liking ratings across all of the sucrose solutions are compared to a moderate liking value of 50 , and SL were classified as those having a mean value $>50$ and SD as having a mean value $<50$. Additionally, liking ratings of sucrose solutions were treated by twoway ANOVA (sucrose concentration and sweet liker status) followed by a multiple pairwise comparison tests (Tukey's HSD) at a significance level of $5 \%$.

Significance of the forced choice RjT paired tests was calculated using the binomial expansion (Diff Test V2.00, 2002 A.W. MacRae), where in each pair the proportion of subjects preferring the control (lowest sucrose level) was compared to the chance probability in a paired test of 0.5 . In addition, to estimate the group rejection thresholds $(\mathrm{RjT})$ the proportion of responses (preference for higher sucrose concentration) were plotted against the log of concentration. Where the data points approximated a straight line a linear model was fitted. The point at which the proportion preferring the higher concentration fell below $50 \%$ was calculated from the linear model, as well as the point at which the proportion preferring the lower concentration reached $75 \%$ (the chance corrected probability for 2AFC tasks) (Lawless, 2010). LMS logged data of perceived sweetness intensity were analyzed by three-way ANOVA with food matrix, sucrose concentration and sweet liker status as treatment effects. FFQ data were analyzed by FETA software (University of Cambridge, UK, FFQ entry and processing program). Nonparametric tests (MannWhitney tests) were used to test for significant differences between SD and SL 
dietary intakes.

Unless otherwise stated, all statistical analysis was carried out using XLStat software (version 2012.1.01, Addinsoft, Paris, France)

\section{Results}

In the results below the sugar content of the samples is referred to as sucrose for simplicity although the samples comprised natural sugars from the orange juice in addition to the added sucrose. The level 1 sample was produced from orange juice with water giving a total sugar content of $33.3 \mathrm{~g} / \mathrm{L}$. The sugar composition of orange juice is approximately 1:1:2 of glucose: fructose: sucrose. Accounting for the difference in relative sweetness of these sugars (approximately 0.74: $1.17: 1.0$ respectively; (Joesten, Hogg, \& Castellion, 2007)) then 33.3 g sugars would be equivalent to approximately $33 \mathrm{~g}$ sucrose in sweetness. We considered this difference minor, and at all high levels of sugar the addition was simply sucrose.

\subsection{Sweet liker status tests}

The categories of SL and SD were determined by two methods. Using cluster analysis (AHC) 13 participants identified as SL (36\%), whereas the other 23 were classified as SD (64\%). For SL, the liking of the aqueous sucrose solutions increased with increasing concentrations of sucrose; however, for SD their liking reduced with increasing concentrations of added sucrose above $6 \%(w / v)$ (Figure 1). In addition, SL and SD were also classified by comparing their average liking of all of the solutions to a moderate liking value of 50 . Using this method, 19 people were classified as SL (53\%), whilst the other 17 consumers were classified as SD (47\%). All of the SL identified by the AHC method were classified as SL by the average liking above mid-point method, however 6 participants identified as SD by the AHC method were characterised as SL by the latter method. The mean liking ratings of these 6 participants was predominantly just above the threshold value of 50 (mean 55.3 compared to mean for other SL of 63.9 and for SD of 42.0). The participant in this group with the highest mean liking (65.2) clearly liked the lower sucrose samples more than the higher sucrose samples (liking ratings of 78.5 and 75.0 for 3 and $6 \%$ sucrose compared to 57.5 and 51.5 for $24 \%$ and $36 \%$ sucrose). The AHC classification was preferred in this study (see discussion). 
282 Considering the whole group's sucrose liking scores, there was no significant difference between the five sucrose solutions $(p=0.287)$, due to the high scores given to samples with high sucrose concentration by SL and the converse by SD. However, there was a significant difference in liking ratings between the groups $(p<0.0001)$ and a significant interaction between the liker group and the sucrose $380 \mathrm{~g} / \mathrm{L}$ sucrose (level 7) ( $p=0.047)$. A rejection threshold was not reached for $\mathrm{SL}$, although a higher proportion of participants in this group was needed to reach the significance criteria as there were fewer people in this group $(n=13)$. The proportion of people preferring the higher concentration of sucrose to the least sweet control was generally higher in the SL group compared to the SD group (Figure 1) until the sucrose concentration was above $380 \mathrm{~g} / \mathrm{L}$ (level 7, log value 2.6 on Figure 2) where in both cases the proportion preferring the sweeter sample was less than 0.5. Using the linear regression equation it was estimated that the point at which the proportion 
of population preferring the higher concentration fell below $50 \%$ would be $279 \mathrm{~g} / \mathrm{L}$ for $\mathrm{SL}$ and $178 \mathrm{~g} / \mathrm{L}$ for SD; however the concentration at which $75 \%$ of the population preferred the lower concentration would exceed the levels tested at $677 \mathrm{~g} / \mathrm{L}$ for SL and $590 \mathrm{~g} / \mathrm{L}$ for SD.

In orange jelly, a RjT was not reached for either the SD or SL group; hence the RjT for sweetness in jelly was higher than $854 \mathrm{~g} / \mathrm{L}$ (L9). Although RjT for jelly was not detected, the proportion preferring sweeter samples in each pair was significantly higher for SL compared to SD ( $p=0.022$, Wilcoxon signed rank test) (Figure 3).

\subsection{Sweetness intensity}

The LMS scale was used to evaluate the sweetness intensity of the jelly and juice samples. As the sucrose concentration increased, the mean intensity score of participants in both SL and SD increased (Figure 4). The difference between the samples was significant overall $(p<0.0001)$. Across matrix and liker category, there was no significant difference between $L 1$ and 2 ( 33.3 and $50 \mathrm{~g} / \mathrm{L}$ sucrose), and these were significantly less sweet than levels 3,4 and 5 (75, 113 and $169 \mathrm{~g} / \mathrm{L})$ which, in turn, were all significantly different from each other and significantly lower than levels $6,7,8$ and $9(253,380,569$ and $854 \mathrm{~g} / \mathrm{L})$. The four highest concentrations were not significantly different from each other.

There was a distinct matrix effect on sweetness. As can be seen in Figure 4, sweetness ratings were significantly lower in jelly than in juice $(p<0.0001)$. This is likely to have caused the differences in $\mathrm{Rj} T$ which were reached in the liquid but not in the semi-solid. Overall, there was a significant overall difference in intensity ratings between SD and SL $(p=0.001)$. However, there was no significant difference between $\mathrm{SD}$ and $\mathrm{SL}$ in their mean ratings within either the juice or the jelly matrix for any individual sucrose level. As shown in Figure 4, the rated intensity of sweetness perception in juice was very similar between SD and SL, whereas in jelly the mean ratings of the SD were higher than the SL for a number of samples but these differences were not significant.

The relationship between $\log _{10}$ perceived intensity versus $\log _{10}$ sucrose concentration was approximately linear in all cases. Within the juice the slope (exponent) values for 
SD and SL were 0.81 and $0.75\left(R^{2}=0.97\right.$ and 0.95 , respectively), respectively, whereas in jelly these values for $S D$ and $S L$ were 0.69 and 0.73 (both $R^{2}=0.95$ ). Thus, the exponents were very similar in all cases indicating that although the intensity of perception of sucrose may vary with the matrix (liquid juice versus semisolid jelly), the rate of increase in sweetness perception with increasing concentration was very similar between both food matrices and very similar for SL and SD (Figure 4).

\subsection{Dietary habits}

Data in the FFQ was used to record total carbohydrate and sugar intake from the participants (Table 1). Although the self-reported mean intake values for total sugars and sucrose were higher in the SL group, the differences were not significant.

Regarding sugar intake as a percentage of total energy intake, the mean was higher for the SL compared to the SD ( $25 \%$ compared to $22 \%$ ); although this difference was not significant $(p=0.087)$.

\section{Discussion}

The rating method used to classify SL and SD was the same in this study as in other recent studies (Holt et al., 2000), whereas previous studies used the Monell forced choice paired comparison method (Mennella et al., 2011) but with the same sucrose concentrations. It was recently demonstrated by Kim et al., 2014 that the patterns of sweet liking determined by the rating method could be confirmed by the Monell method. The proportion of SL in the present study (36\% when determined by the AHC method, $53 \%$ when determined by the average liking above mid-point method) was higher than in the Holt et al. (2000) study where they found $12 \%$ of Australians and Malaysians to be SL, but lower than the Yeomans et al., 2009 study where $60 \%$ of UK students were found to be SL and the Kim et al., 2014 study where $50 \%$ of Koreans were classified as SL. However, classification methods differed in the studies. Yeomans et al., 2009 used the average liking above mid-point method, resulting in a similar proportion of $S L$ as in our study when we classified by this method. However, using this approach we concluded that participants were easily misclassified. For instance, one participant's average liking score was more than 50 , but his liking score decreased with increasing sample sucrose concentration. Although such discrepancies could be resolved to some extent by normalising 
individual results before classification, we found the cluster analysis by AHC to be the most successful method of grouping SL and SD. Holt et al., 2000 classified through the shape of reponse curve, with SL giving progressively higher liking scores as sucrose increased and SD displaying either an optimum concentration (4-8\% sucrose) after which liking decreased, or a continual decline in liking ratings with sucrose concentration. This is rather similar to the use of cluster analysis in our study and of the study by Kim et al., 2014 which identified 3 clusters, the SL cluster increased their liking ratings with concentration and the other 2 clusters had an optimum sucrose concentration within aquous solutions of approximately $12 \%$ sucrose.

In Mennella et al., 2011 the most preferred sucrose concentration by adults was $14.4 \% \mathrm{w} / \mathrm{v}$. In our study, although there was no significant difference in the liking of the different sucrose concentrations by the group as a whole, the sucrose concentration most liked by SL was $14.4 \% \mathrm{w} / \mathrm{v}$, while SD gave highest mean liking scores to the $6 \% \mathrm{w} / \mathrm{v}$ sucrose solution. These results suggest that preferred sucrose concentration should not be averaged across a group but that sweet liker status should be taken into consideration. This can be inferred from much earlier studies by Rose Marie Pangborn; firstly in a study of sucrose in coffee subjects were classified into four groups according to their hedonic response curve (liking either decreased, increased, reached an optimum or was unaffected by increasing sucrose concentration) (Lundgren et al., 1978), and in a second study where differences in liking for sweetness level in lemonade were found to correlate to intake of sweet foods (Pangborn \& Giovanni, 1984).

One limitation of the method used by ourselves and others is that classification based on responses in aqueous solutions may not relate to liking of sweetness in real foods. Indeed in the Holt study, some consumers classified as SD scored food samples with increasing sucrose levels higher in liking than some individuals classified as SL. In the Kim et al. 2014 study although the 2 clusters defined as SD reached an optimum sucrose concentration for liking in aqueous solution (at 12 or $24 \%$ sucrose), one of these clusters (31\%) continued to increase their liking with sucrose concentrations up to $36 \%$ in beverages. Relating this to the present study, although our SD group reached an optimum sucrose concentration for liking in 
aqueous solutions at a lower level $(6 \% \mathrm{w} / \mathrm{v}$, Figure 1$)$, their rejection of orange juice samples at $380 \mathrm{~g} / \mathrm{L}$ sucrose $(38 \%)$ is very similar to the findings of Kim et al. (2014) where liking for sucrose in a beverage reduced at $36 \%$ sucrose. However, classification of SD and SL from aqueous sucrose solutions did not predict participants' RjT for sucrose in orange jelly where the sucrose concentrations were perceived to be far less intense (Figure 4) and RjT were not reached (Figure 3).

Taste and food preferences have previously been shown to have an important role in RjT. Studies by Harwood classified participants as bitter likers (preferring dark chocolate) and bitter dislikers (preferring milk chocolate). Compared to bitter dislikers, bitter likers had a higher RjT for the bitter tastant sucrose octaacetate within chocolate milk (Harwood et al., 2012a). Moreover, RjT of bitter dislikers was lower than for bitter likers in solid chocolate (Harwood et al., 2012b). Similarly in the present study, within orange juice SD had a lower RjT for sucrose $(380 \mathrm{~g} / \mathrm{L})$ than $\mathrm{SL}$ where the exact RjT was not determined, but was $>380 \mathrm{~g} / \mathrm{L}$ ). Within orange juice this confirmed the hypothesis that SL would have higher RjT for sucrose that SD. However, although higher sweet levels were used in the jelly than in the juice, the highest sucrose concentration ( $854 \mathrm{~g} / \mathrm{L}$ ) in jelly test failed to reach the RjT. The matrix effected the perception of sweet intensity, as shown in the perceived intensity (LMS) results and, this led to a difference in the RjT. It well known that viscosity effects perception of both taste and aroma and the possible causative mechanism were discussed in a review by (Cook, Hollowood, Linforth, \& Taylor, 2005). They concluded that sweetness perception decreased with viscosity which fully supports the findings of the current study that perceived sweet intensity was substantially and significantly lower in jelly compared to juice. In line with our findings, Holt et al., 2000 concluded that perception of sweet intensity and the sucrose addition levels which led to optimum liking were food-specific. They found sweetness in biscuits was lower than in orange juice at the same added sugar levels and that the most liked sugar level was higher in biscuits than in orange juice. However, the sweetness of orange juice and biscuits would be moderated by other tastants in the foods, particularly acidity and fat respectively, so the differences between the food types were not just due the physical properties of the matrix. In the current study, although a significant $\mathrm{RjT}$ in orange juice was not reached until $380 \mathrm{~g} / \mathrm{L}$, the proportion of people preferring the sweeter sample started to decrease after $75 \mathrm{~g} / \mathrm{L}$ (or $7.5 \% \mathrm{w} / \mathrm{v}$ ) which is in line 
with the Holt study finding.

Although SD and SL had significantly different mean ratings of perceived sweet intensity from the LMS tests, the actual differences between SL and SD were very small and not significant within one matrix at any specific sugar concentration. From this we might conclude that preference for sweet foods did not influence sweet taste intensity perception, or vice versa. However, we also recognize that we used the LMS scale for measuring perceived intensity rather than the gLMS scale and, therefore as highlighted by (Bartoshuk et al., 2006) the perceived intensities denoted by the semantic labels may vary with the participants experience of the tastant. Following this argument, SD perception of "strong sweet taste" might be at a lower sucrose concentration than for SL, so SD might be expected to rate equivalent sucrose concentrations higher on the LMS scale than SL. However, this was not the case, the overall mean sweet intensity for SD (26.4) was lower than for SL (29.9). So accounting for the possible difference in experience of sweet taste, the difference we found between SD and SL in rated intensity might be slightly less if we had measured it on a stimulus generic gLMS scale. However, this would have led to the same overall conclusion that the differences in perceived sweetness between SD and SL was very small and could not account for their differences in sweet liking or RjT.

Similarly, in the previous chocolate milk study, bitter likers and dislikers differed in their RjT whilst their bitter detection thresholds for the same bitter compound in the chocolate milk were not statistically different (Harwood et al., 2012a). This suggests that the ability to detect bitterness did not directly influence the consumers' acceptability of bitter taste. It would, therefore appear that liking and RjT for both sweetness and bitterness are both not directly influenced by consumer sensitivity to these tastants, at least within the food matrices and parameters of these two studies.

The Steven's power functions for sweetness in orange juice and orange jelly showed slightly decelerating relationships with exponents approximating 0.75 . A similar exponent of 0.78 has been reported in the literature for sweetness perception of sucrose in water, where an LMS scale was used by 20 subjects to rate sweetness intensity of aqueous sugar solutions (Green, Shaffer, \& Gilmore, 1993). In this former study the concentration range was slightly lower than in the current study (from 0.05 
to $0.8 \mathrm{M}$, or 17 to $274 \mathrm{~g} / \mathrm{L}$, in water compared to 33.3 to $569 \mathrm{~g} / \mathrm{L}$ in orange juice); however unlike in water, the sweet perception in orange juice may have been suppressed by acidity in the juice. It has previously been demonstrated that suppression within binary taste mixtures decreases the slope of the psychophysical curve and reduces the exponent (Keast \& Breslin, 2003); hence lower exponents might have been expected in this study for sweetness perception within the orange matrices. The Green study reported identical exponent values whether rated was done by the LMS scale or magnitude estimation (ME); however an earlier study reported a higher exponent of 1.13 using ME (Kroeze, 1976). This earlier study used a lower maximum sucrose concentration ( $195 \mathrm{~g} / \mathrm{L}$ ) which may explain the higher exponent as the increase in perceived intensity with increasing concentration would not have started to plateau. In the current study, if the exponent is calculated from only the data from 33.3 to $168.7 \mathrm{~g} / \mathrm{L}$ sucrose in orange juice, the value increases to 1.08 for SD and 0.91 for SL.

Results from the current study suggest that sweet liker status is not related to differences in intensity of sweet perception, this may imply that differences in sweet liking are a learned behavior rather than a physiological taste response. A similar conclusion was drawn from an earlier study within coffee where ability to discriminate among sucrose levels and degree of liking for sucrose levels in coffee were found to be independent behavioral responses (Lundgren et al., 1978). This appears encouraging as it implies that sweet liker status could be modified; although it does not rule out inter-individual differences in physiological feedback. However, a more recent study by Wise et al., 2016 investigated the effect of a 3 month low sugar diet on sweetness perception and liking. They found that sweetness intensity was rated significantly higher following the low sugar diet, again encouraging, and yet this did not lead to a change in sweet liking. In our study, the small trend in difference for self-reported sugar intake between the SL and SD was also not promising. Both groups had over $20 \%$ of their energy intake as sugars, far in excess of dietary guidelines which recommend that daily intake of sugars should be less than $10 \%$ of total energy intake, with a further reduction to less than $5 \%$ providing additional health benefits (WHO, 2015).

\section{Conclusions}


This study concluded that the RjT method used in previous studies to determine rejection thresholds for objectionable flavours could be successfully used to detect RjT for desirable flavours, in this case sweetness. RjT for sweetness was influenced by liking of sweet taste and within orange juice sweet likers had a higher RjT for sucrose than sweet dislikers. Perceived sweetness was much lower in a semi-solid jelly than in a liquid juice at equivalent sucrose concentration and, hence, RjT in jelly were not reached. Although there was a statistically significant difference in perceived sweet intensity between SL and SD, the extent of difference between the two groups was very minor. It was therefore inferred that differences in sweet liker status and sucrose RjT were influenced by factors other than perceptual differences in sweetness. Hence, future studies to investigate the effects of repeated exposure to low-sweetness as well as low-sugar diets on sweet liking and sucrose RjT are recommended. Larger studies with a broader spectrum of consumers are needed to determine whether sweet liker status has a significant impact on dietary intake of sugars and BMI.

\section{Acknowledgments}

All volunteers in the study are thanked for their time. Compusense are acknowledged for the provision of the software that enabled the study data collection. The authors would like to acknowledge the contribution of the staff of the EPIC-Norfolk Study for their software to analyse of the food frequency questionnaire diaries. EPIC-Norfolk is supported by the Medical Research Council program grants (G0401527,G1000143) and Cancer Research UK program grant (C864/A8257).

References:

Bartoshuk, L. M., Duffy, V. B., Hayes, J. E., Moskowitz, H. R., \& Snyder, D. J. (2006). Psychophysics of sweet and fat perception in obesity: problems, solutions and new perspectives. Philosophical Transactions of the Royal Society BBiological Sciences, 361(1471), 1137-1148.

Berridge, K. C. (2003). Pleasures of the brain. Brain and Cognition, 52(1), 106-128.

Cicerale, S., Riddell, L. J., \& Keast, R. S. J. (2012). The Association between Perceived Sweetness Intensity and Dietary Intake in Young Adults. Journal of Food Science, 77(1), H31-H35.

Cook, D. J., Hollowood, T. A., Linforth, R. S. T., \& Taylor, A. J. (2005). Correlating instrumental measurements of texture and flavour release with human perception. International Journal of Food Science and Technology, 40(6), 631641. 
Drewnowski, A., Mennella, J. A., Johnson, S. L., \& Bellisle, F. (2012). Sweetness and Food Preference. Journal of Nutrition, 142(6), 1142S-1148S.

EPIC (2015). European Prospective Investigation into Cancer and Nutrition. http://epic.iarc.frl [Accessed on 12/6/2015]..

Green, B. G., Shaffer, G. S., \& Gilmore, M. M. (1993). Derivation and evaluation of a semantic scale of oral sensation magnitude with apparent ratio properties. Chemical Senses, 18(6), 683-702.

Harwood, M. L., Loquasto, J. R., Roberts, R. F., Ziegler, G. R., \& Hayes, J. E. (2013). Explaining tolerance for bitterness in chocolate ice cream using solid chocolate preferences. Journal of Dairy Science, 96(8), 4938-4944.

Harwood, M. L., Ziegler, G. R., \& Hayes, J. E. (2012a). Rejection thresholds in chocolate milk: evidence for segmentation. Food Quality and Preference, 26(1), 128-133.

Harwood, M. L., Ziegler, G. R., \& Hayes, J. E. (2012b). Rejection thresholds in solid chocolate-flavored compound coating. Journal of Food Science, 77(10), S390-S393.

Harwood, M. L., Ziegler, G. R., \& Hayes, J. E. (2013). Tolerance for High Flavanol Cocoa Powder in Semisweet Chocolate. Nutrients, 5(6), 2258-2267.

Hayes, J. E., \& Duffy, V. B. (2008). Oral sensory phenotype identifies level of sugar and fat required for maximal liking. Physiology \& Behavior, 95(1-2), 77-87.

Holt, S. H. A., Cobiac, L., Beaumont-Smith, N. E., Easton, K., \& Best, D. J. (2000). Dietary habits and the perception and liking of sweetness among Australian and Malaysian students: A cross-cultural study. Food Quality and Preference, 11(4), 299-312.

Ji-Yoon, K., Prescott, J., \& Kwang-Ok, K. (2014). Patterns of sweet liking in sucrose solutions and beverages. Food Quality and Preference, 36, 96-103.

Joesten, M. D., Hogg, J. L., \& Castellion, M. E. (2007). Sweeteness Relative to Sucrose (table, pg 359), In :The World of Chemistry: Essentials: Belmont, California: Thomson Brooks/Cole.

Keast, S. J. R., \& Breslin, P. A. S. (2003). An overview of binary taste-taste interactions. Food Quality and Preference, 14(2), 111-124.

Kim, J.-Y., Prescott, J., \& Kim, K.-O. (2014). Patterns of sweet liking in sucrose solutions and beverages. Food Quality and Preference, 36, 96-103.

Kroeze, J. H. A. (1976). Exponential values of psychophysical power function for sucrose obtained by 2 different estimation methods. Chemical Senses \& Flavour, 2(1), 39-43.

Lawless, H. T. (2010). A simple alternative analysis for threshold data detemrined by ascending forced-choice methods of limits. Journal of Sensory Studies, 25(3), 332-346.

Lundgren, B., Jonsson, B., Pangborn, R. M., Sontag, A. M., Barylkopikielna, N., Pietrzak, E., et al. (1978). Taste discrimination vs hedonic response to sucrose in coffee beverage. An interlaboratory study. Chemical Senses \& Flavour, 3(3), 249-265.

Mennella, J. A., Lukasewycz, L. D., Griffith, J. W., \& Beauchamp, G. K. (2011). Evaluation of the Monell forced-choice, paired-comparison tracking procedure for determining sweet taste preferences across the lifespan. Chemical Senses, 36(4), 345-355.

Moskowitz, H. R. (1978). Psychological correlates of sugar consumption. Journal of Food Quality, 2(1), 27-40.

Pangborn, R. M. (1970). Individual variation in affective responses to taste stimuli. 
Psychonomic Science, 21(2), 125-126.

Pangborn, R. M., \& Giovanni, M. E. (1984). Dietary-intake of sweet foods and of dairy fats and resultant gustatory responses to sugar in lemonade and to fat in milk. Appetite, 5(4), 317-327.

Prescott, J., Norris, L., Kunst, M., \& Kim, S. (2005). Estimating a "consumer rejection threshold" for cork taint in white wine. Food Quality and Preference, 16(4), 345-349.

Saliba, A. J., Bullock, J., \& Hardie, W. J. (2009). Consumer rejection threshold for 1,8-cineole (eucalyptol) in Australian red wine. Food Quality and Preference, 20(7), 500-504.

WHO (2015). World Health Authority Guideline: Sugars intake for adults and children. WHO Press, Geneva, Switzerland. ISBN 9789241549028.

Wise, P. M., Nattress, L., Flammer, L. J., \& Beauchamp, G. K. (2016). Reduced dietary intake of simple sugars alters perceived sweet taste intensity but not perceived pleasantness. American Journal of Clinical Nutrition, 103(1), 50-60.

Yeomans, M. R., Prescott, J., \& Gould, N. J. (2009). Acquired hedonic and sensory characteristics of odours: Influence of sweet liker and propylthiouracil taster status. Quarterly Journal of Experimental Psychology, 62(8), 1648-1664.

Yoo, Y. J., Saliba, A. J., Prenzler, P. D., \& Ryan, D. (2012). Total phenolic content, antioxidant activity, and cross-cultural consumer rejection threshold in white and red wines functionally enhanced with catechin-rich extracts. Journal of Agricultural and Food Chemistry, 60(1), 388-393. 


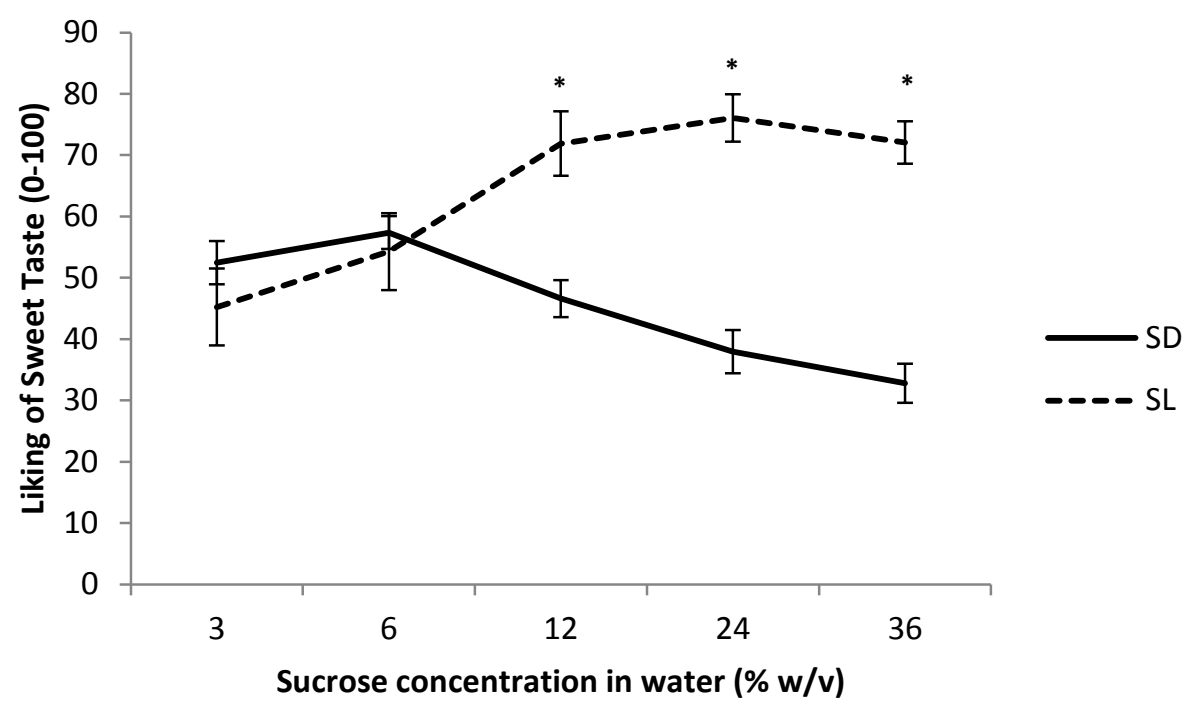

633

Figure 1. The mean liking of sweet taste for the sucrose solutions for sweet likers and dislikers $(n=36)$. SL and SD groupings determined agglomerative hierarchical cluster analysis. Error bars represent $+/$ - standard error of the mean. Significant differences in ratings between SL and SD indicated by * $(p<0.05)$.

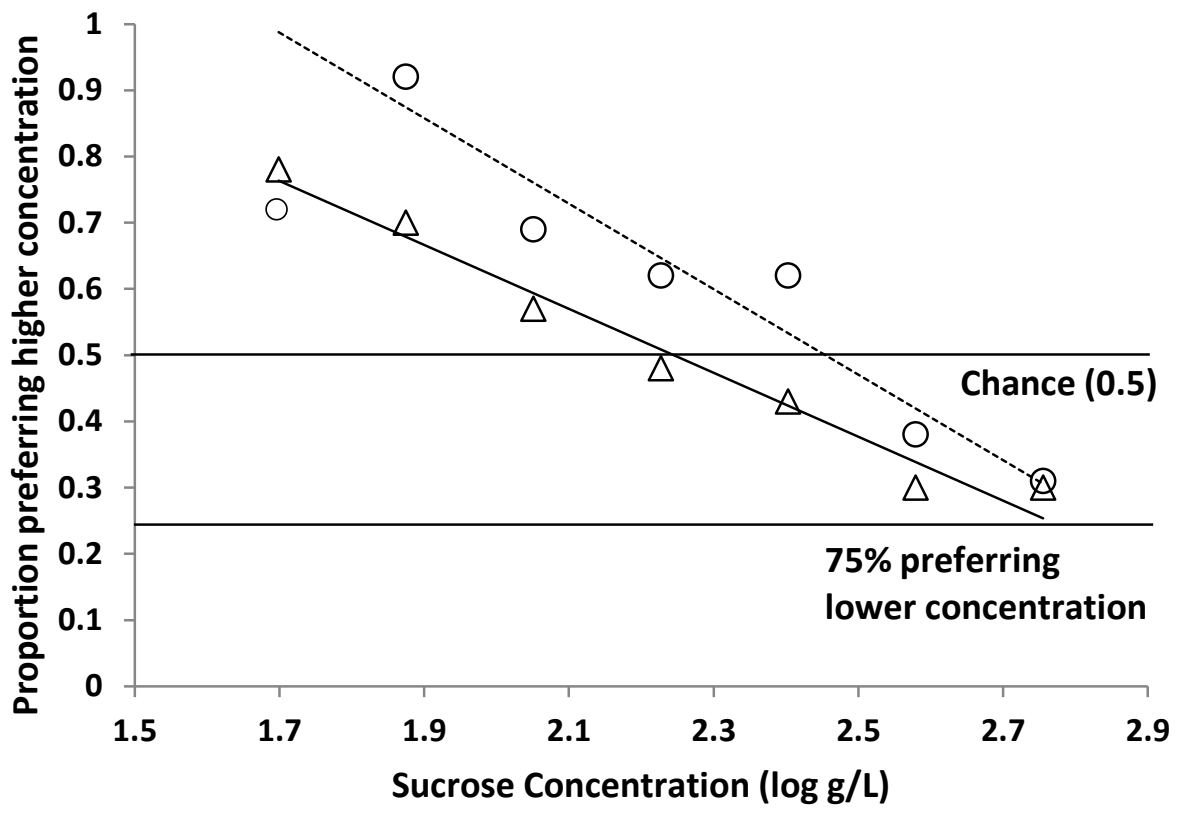

OSweet Likers (SL); y = -0.65x + 2.09; $R^{2}=0.93$

$\triangle$ Sweet Dislikers (SD); $y=-0.48 x+1.58 ; R^{2}=0.97$

Figure 2. Proportion of participants preferring the orange juice containing the higher concentration of sucrose

(note : the first value for SL was removed in order to fit the regression line) 


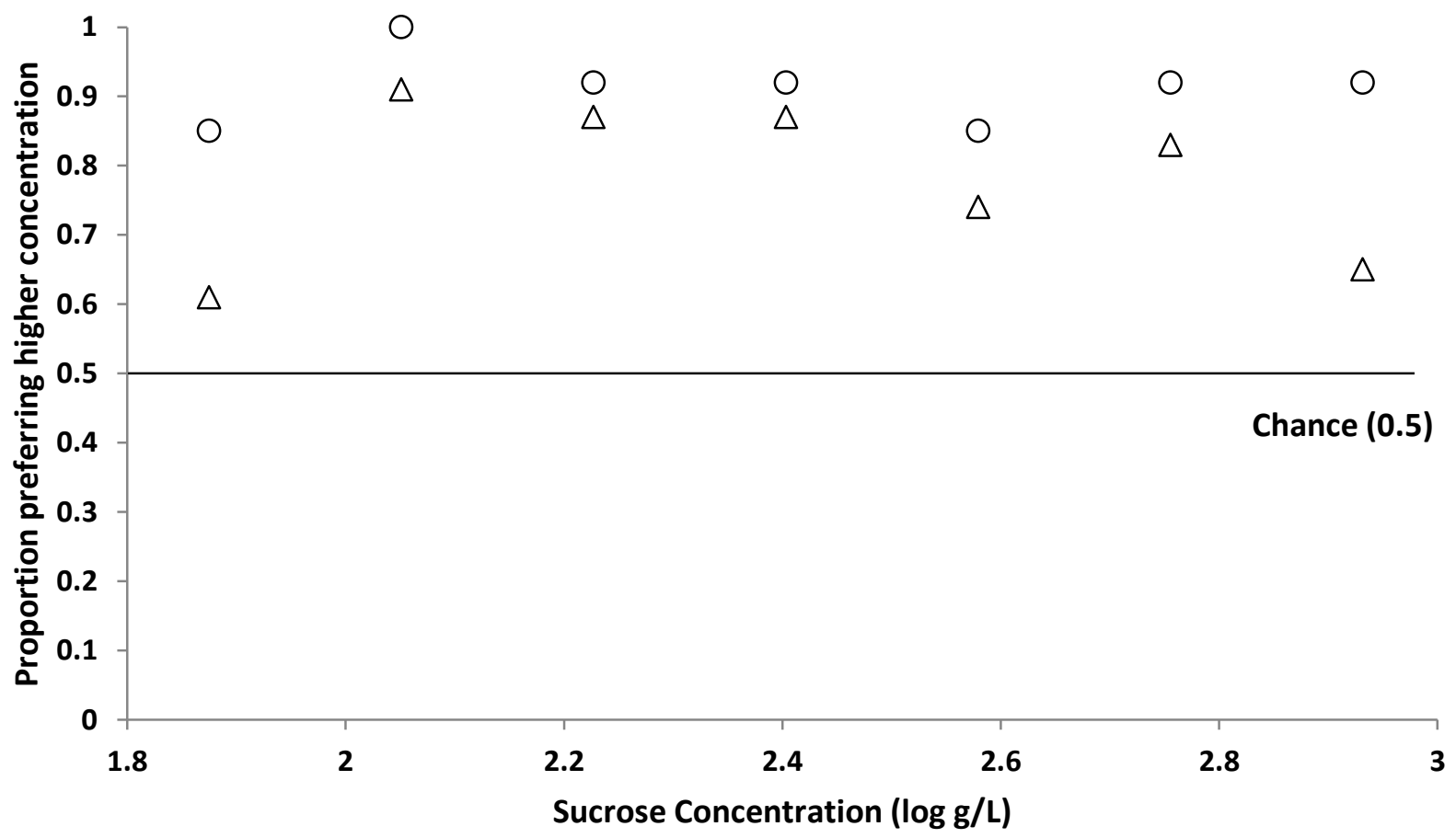

OSweet Likers (SL) $\triangle$ Sweet Dislikers (SD)

Figure 3. Proportion of participants preferring the orange jelly containing the higher concentration sucrose

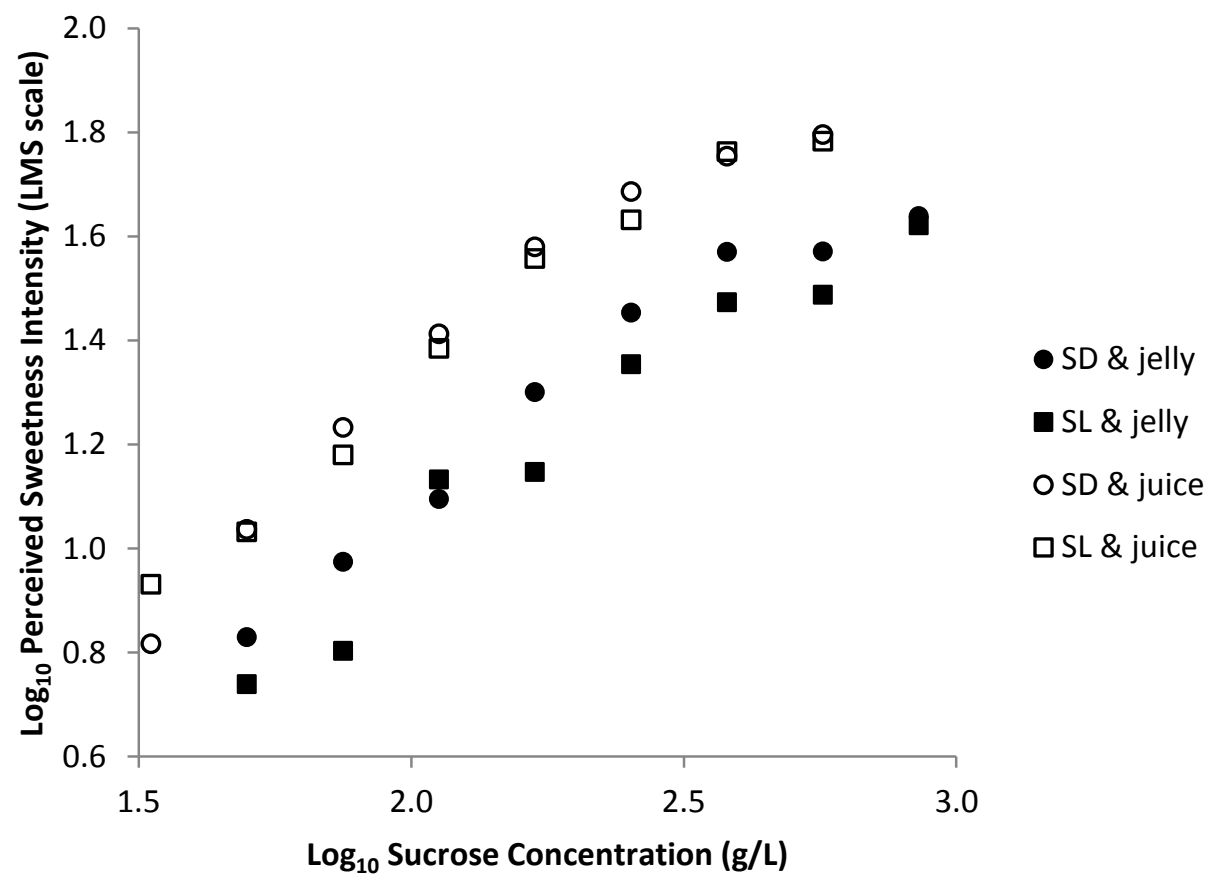

Figure 4. Sweetness intensity as a function of sucrose concentration (Log-Log data) in orange juice and jelly 


\begin{tabular}{|c|c|c|c|}
\hline & Total $^{\mathrm{a}}$ & $\begin{array}{l}\text { Sweet } \\
\text { Likers }\end{array}$ & Sweet Dislikers \\
\hline Female $\mathrm{n}(\%)$ & $23(66)$ & $9(75)$ & $14(61)$ \\
\hline Male n (\%) & $12(34)$ & $3(25)$ & $9(39)$ \\
\hline Age years range (median) & $18-50(26)$ & $20-50(26)$ & $18-50(25)$ \\
\hline BMI $\mathrm{kg} / \mathrm{m}^{2}$ range (median) & $17-29(22)$ & $17-29(24)$ & $19-25(21)$ \\
\hline \multicolumn{4}{|l|}{ Ethnicity n (\%): } \\
\hline Caucasian & $13(37)$ & $4(33)$ & $9(39)$ \\
\hline Chinese & $15(43)$ & $4(33)$ & $11(48)$ \\
\hline Other Asian & $7(20)$ & $4(33)$ & $3(13)$ \\
\hline
\end{tabular}

Daily intake from FFQ, mean \pm standard deviation:

Total Carbohydrate (g / day)

Total Sugars (g / day)

$307 \pm 240 \quad 272 \pm 128$

Sucrose (g / day)

$150 \pm 89 \quad 126 \pm 66$

Sugars as \% Energy Intake

$61 \pm 38 \quad 56 \pm 34$

$25 \pm 6.3$

$22 \pm 3.9$

657

${ }^{a}$ Of 36 people in the study, 1 participant denied demographic information

658

659 